\title{
Correction to: Transmissible a-synuclein seeding activity in brain and stomach of patients with Parkinson's disease
}

\author{
Achim Thomzig ${ }^{1} \cdot$ Katja Wagenführ $^{1,2} \cdot$ Phillip Pinder $^{1} \cdot$ Marion Joncic $^{1} \cdot$ Walter J. Schulz-Schaeffer ${ }^{3}$. \\ Michael Beekes ${ }^{1}$ (1)
}

Published online: 6 May 2021

(c) The Author(s) 2021

\section{Correction to: Acta Neuropathologica https://doi.org/10.1007/s00401-021-02312-4}

In the original publication, Table 2 contains inadvertent inconsistencies with respect to the bank vole bioassay raw data reported in Supplementary Table 1 (Online Resource), and the findings on localized SDC/DN pathology reported for PStH A erroneously represented the ratio of animals with and without localized SDC/DN pathology, instead of correctly the number of animals with localized SDC/DN pathology per number of examined animals. Data provided in the 4th, 8th, 11th and 12th row of Table 2 therefore need to be duly corrected as follows.

The original article can be found online at https://doi.org/10.1007/ s00401-021-02312-4.

Michael Beekes

BeekesM@rki.de

Achim Thomzig

ThomzigA@ rki.de

Katja Wagenführ

Katja.Wagenfuehr@lageso.berlin.de

Phillip Pinder

phillip.pinder@arcor.de

Marion Joncic

JoncicM@rki.de

Walter J. Schulz-Schaeffer

Walter.Schulz-Schaeffer@uks.eu

1 Prion and Prionoid Research Unit, ZBS 6-Proteomics and Spectroscopy, ZBS - Centre for Biological Threats and Special Pathogens, Robert Koch Institute, Nordufer 20, 13353 Berlin, Germany

2 Present Address: State Office for Health and Social Affairs (LAGeSo), Berlin, Germany

3 Institute of Neuropathology, Faculty of Medicine, Saarland University, Homburg, Germany
Fourth row (male, PStH A): median age - 599 (instead of 595); mean age - 595 (instead of 599); localized SDC/DN pathology-3/7 (instead of 3/4).

Eighth row (female, PStH A): median incubation period - 546 (instead of 541); mean incubation period - 541 (instead of 546); median age - 599 (instead of 595); mean age-595 (instead of 599); localized SDC/DN pathology-4/7 (instead of 4/3).

Eleventh row (male and female, PBH B): mean incubation period-554 (instead of 550); mean age - 606 (instead of 602).

Twelfth row (male and female, PStH A): median age599 (instead of 595); mean age - 595 (instead of 599); localized SDC/DN pathology-7/14 (instead of 7/7).

In addition, the sentence "Of these animals, four females developed localized SDC/DN pathology of sub-phenotype L+ (Fig. 6c)" in the second-last paragraph of the results section should correctly read "Of these animals, three females and one male developed localized SDC/DN pathology of sub-phenotype L+ (Fig. 6c)".

These corrections do not affect any material findings, claims or conclusions of the study.

Open Access This article is licensed under a Creative Commons Attribution 4.0 International License, which permits use, sharing, adaptation, distribution and reproduction in any medium or format, as long as you give appropriate credit to the original author(s) and the source, provide a link to the Creative Commons licence, and indicate if changes were made. The images or other third party material in this article are included in the article's Creative Commons licence, unless indicated otherwise in a credit line to the material. If material is not included in the article's Creative Commons licence and your intended use is not permitted by statutory regulation or exceeds the permitted use, you will need to obtain permission directly from the copyright holder. To view a copy of this licence, visit http://creativecommons.org/licenses/by/4.0/.

Publisher's Note Springer Nature remains neutral with regard to jurisdictional claims in published maps and institutional affiliations. 